\title{
The Approach to Engineering Tasks Composition on Knowledge Portals
}

\author{
R. Novogrudska, L.Globa \\ National Technical University of Ukraine \\ «Kyiv Polytechnic Institute», \\ Information Telecommunication Networks \\ Department \\ Kyiv, Ukraine \\ rinan@ukr.net, lgloba@its.kpi.ua
}

\author{
A.Schill \\ Dresden University of Technology \\ Faculty of Computer Science \\ Dresden, Germany \\ alexander.schill@tu-dresden.de
}

\begin{abstract}
The paper presents an approach to engineering tasks composition on engineering knowledge portals. The specific features of engineering tasks are highlighted, their analysis makes the basis for partial engineering tasks integration. The formal algebraic system for engineering tasks composition is proposed, allowing to set the context-independent formal structures for engineering tasks elements' description. The method of engineering tasks composition is developed that allows to integrate partial calculation tasks into general calculation tasks on engineering portals, performed on user request demand. The real world scenario «Calculation of the strength for the power components of magnetic systems» is represented, approving the applicability and efficiency of proposed approach.
\end{abstract}

Keywords: engineering task, knowledge portals, algebraic model, composition, parameters.

\section{The Approach to Engineering Tasks Composition on Knowledge Portals}

\author{
R. Novogrudska, L.Globa \\ National Technical University of \\ Ukraine «Kyiv Polytechnic Institute», \\ Information Telecommunication Net- \\ works Department \\ Kyiv, Ukraine \\ rinan@ukr.net,1globa@its.kpi.ua
}

\author{
A.Schill \\ Dresden University of Technology \\ Faculty of Computer Science \\ Dresden, Germany \\ alexander.schill@tu-dresden.de
}

\begin{abstract}
The paper presents an approach to engineering tasks composition on engineering knowledge portals. The specific features of engineering tasks are highlighted, their analysis makes the basis for partial engineering tasks integration. The formal algebraic system for engineering tasks composition is proposed, allowing to set the context-independent formal structures for engineering tasks elements' description. The method of engineering tasks composition is developed that allows to integrate partial calculation tasks into general calculation tasks on engineering portals, performed on user request demand. The real world scenario «Calculation of the strength for the power components of magnetic systems» is represented, approving the applicability and efficiency of proposed approach.
\end{abstract}

Keywords: engineering task, knowledge portals, algebraic model, composition, parameters.

\section{INTRODUCTION}

Research results of independent agencies Gartner Group and Real Story Group [1] for 2011-2012 show that one of the major trends in the development of content management is moving from simple structure web-sites to large integrated portals of corporate knowledge.
These portals contain a description of the different scientific, engineering or industrial tasks, information about methods of solving or providing services to implement them. They also provide users with navigation search and extension of the displayed content with required detailing.

Integration of data and computing resources by using portals solutions that contain links to geographically distributed resources, allows not only to access the information resources, stored in their databases, but also, using data, knowledge and services to implement certain tasks. When the number of links to different information and computing resources becomes large enough, the efficient management of information and computing processes becomes almost impossible.

Knowledge portal is an integrated portal designed to provide meaningful access to structured data and portal knowledge. Information, concentrated in knowledge portals' environment, makes their usage very common. Thus, technically, knowledge portals should provide integration and collaboration of services, databases and knowledge bases, information storages and computing resources. 
Knowledge portals can be devoted to different subject domains. The knowledge portals that represent information on engineering subject domain (radio engineering, chemical industry, strength of materials, aerospace industry, materiology, casting manufacturing, etc.) are called engineering knowledge portals. Demand of the engineering knowledge portals depends on the ability to perform online different engineering tasks by the end user. The environment of such portals allocates great amount of different engineering tasks (ETs). This allow portal users to carry out real-time calculations related to the portal subject domain (for example, a portal related to the subject domain strength of materials should allow the user to calculate: the strength of construction, the resistance of construction, the construction element on crack resistance, etc.). For the end user it is necessary to get correct result of ETs in minimal time. These factors are main for estimation of the engineering portal usage efficiency.

The proposed research is aimed at improving the efficiency of engineering tasks implementation on knowledge portals.

The main problem is the lack of a method of engineering tasks composition that will set the sequence of engineering tasks that should be included in complex engineering task on users demand. The use of such method for the engineering knowledge portals designing will ensure the efficiency of engineering tasks performance and improve the automation level of their composition.

Solution of the specified problem requires solving of the next tasks:

- to explore the characteristics of ETs that allow to define the rules by which it is possible to link them,

- to develop an algebraic system that allows to describe the elements of the portal involved in the ETs and their relationships,

- $\quad$ to develop a method of engineering tasks composition for the formation and implementation of complex engineering calculations.

This paper describes a novel approach to knowledge portals engineering tasks composition, allowing to reduce the time of their performance as well as to automate the process of their interaction.

The paper is structured as follows: Section 2 provides the analyses of existing approaches and models that are used for the knowledge portals design, as well as approaches to Webservices composition used for the performance of engineering tasks. Section 3 contains the description of engineering tasks characteristic features. Section 4 describes the algebraic system of engineering tasks. Section 5 describes new method of engineering tasks composition. Section 6 highlights evaluation results, the evaluation has been applied using a real-world scenario within subject domain "Strength of materials". Section 5 concludes the work with a summary and outlook on future work.

\section{BACKGROUND AND RELATED WORKS}

\section{The approaches to portals development}

The typical approach to portals development includes several stages:

1. Conceptual design.

2. Logical design.

3. Physical design.

At each stage the appropriate software environment, products and notation are used. There are specialized software plat- forms that enable the development of Internet-portals. Usually such solutions have already implemented basic functionality. Such solutions include: IBM WebSphere, Oracle Collaboration Suite, SAP NetWeaver Portal, Microsoft Office SharePont Server [2,3,4,5].

Portals designed based on these platforms provide users with the ability of collaborated work. They can be used as the mechanism for analysis and systematization of information or as repository of information, knowledge and document, as well as for storage and execution of web-based software. The users are given the opportunity to manage and interact with information in lists and document libraries using the controls elements that are called Web Parts. The usage of such specialized software platforms provides a integrated platform through which users can interact effectively with other members of the group, find resources, search for experts and corporate information, manage information and workflows, as well as analyze business data to make more informed decisions.

Since the environment of engineering knowledge portal concentrates a large amount of information and software resources, the portal development platform should provide the ability to display these resources in different formats and the ability to integrate the applications for displaying of information resources using the means of workflow formation. Portal's workflow can be considered as means to automate the execution of tasks sequence related to the engineering tasks implementation.

One of the most widespread scientific approaches to the knowledge portals design is an approach that is based on the ontological model usage [6-12]. Portal's structure presentation in the form of ontology allows not only to systematize and organize the information represented on the portal but also to describe the portal informational items and to set the relationship between them [13]. As an ontological model is a model of semantic type, its usage allows to describe directly the semantic coherence and connectivity of the portal's elements. The example of ontological model usage for the knowledge portals in archeology and computational linguistics designing is described in [14]. Thus, ontology allows to describe portal information objects and their information environment but ontology does not solve the problem of engineering tasks integration concentrated in the engineering portals space.

\section{The approaches to Web-services composition}

Web Service Composition is a method to connect different web services that are used for creating high level business architecture by compiling of web services in order to provide functionalities that are not available during design [15]. Consequently, there is a possibility to develop a new functionality by simply reusing of components that are already available but unable to complete a task successfully on their own.

Static and Dynamic WSC are two major types of Web service composition. Dynamic web service composition occurs when user or software application queries for a web service at runtime while static web service composition is performed at the compile time. Dynamic web service composition is less trivial and requires much more work compared to static web service composition.

Various authors classify other WSC approaches. In [16] Static and Dynamic Web Service Composition approaches are grouped into a single approach. This and other possible approaches for composition are listed below:

- $\quad$ Static and Dynamic Composition ;

- Model Driven Service Composition; 
- Declarative Service Composition;

- Automated and Manual Composition;

- Context-based Service Discovery and Composition.

The ability to select and compose heterogeneous Web services over the Web efficiently and effectively at runtime is an important step towards the development of the Web service applications [17]. By utilizing Web services end user is able to create composite services to fulfil the requirement when single service unable to do it.

Most of the approaches related to the Web service composition $[18,19,20,21]$ realized the fact that the prerequisite tasks to generate the composition solution are the service discovery and service selection of the candidate Web services stored in the service repository.

Semantic web services [22,23,24,25] provide an open, extensible, semantic framework for describing and publishing semantic content, improved interoperability, automated service composition, discovery and invocation, access to knowledge on the Internet [26].

$[27,28,29]$ describes discovery and composition issues of Web services in the proposed extended SOA architecture. The concept of automated service discovery and composition process using Semantic Web services is presented.

In agent-based solutions for web-services composition agencies gather QoS data from agents, store, aggregate and present it to agents [30]. Approach that solves these problems is introduced in [31]. Distinctive feature of this approach is utilization of SLA via WS-Agreement [32] during both workflow enactment and workflow analysis stages.

A composite service is a set of individual services that are combined effectively and reused in order to achieve a desired effect. Automatic Web service composition consists of four phases: Discovery, Planning, Selection, and Execution [33]. The first phase involves creating a plan, i.e., sequence of services in desired composition. The plan creation could be manual, semi-automatic, or automatic. The second phase embodies service discovery due to the plan. Planning and discovery are often combined into one step. After discovery of suitable services, the selection phase starts. It embodies a selection of the optimal composition from the available combinations of web services judging on nonfunctional properties like QoS properties. The final phase involves executing the services due to the plan. If some service is not available, another one takes it place. Concerning ET performance on engineering knowledge portals the most time-consuming is the phase of Discovery. As it is in need to choose from ETs variety only those that are to be composed for complex engineering calculation performance.

THE CHARACTERISTIC FEATURES OF ENGINEERING TASKS

This section offers the detailed description of engineering tasks, their peculiarities and types.

All engineering tasks can be divided into 2 types: complex and partial. Complex engineering tasks consist of partial and allow to perform complex engineering calculations. Partial engineering tasks allow only to determine certain feature or parameter and they do not show overall picture of how to perform engineering calculations (for example, for the engineering subject domain "strength of material": complex ET - calculation of the strength of magnetic systems - allows to undertake a comprehensive assessment of the magnetic system on the strength, partial calculations - calculation of resistance, calculation of elasticity - allow to determine that the construc- tion satisfies some partial characteristic) [34,35]. In the process of complex ET formation the composition of partial ETs is held. Thus, to improve the efficiency of ETs performance on the engineering knowledge portals it is necessary to reduce the time of partial ETs composition as well as to automate the process of their composition.

From a technical point of view (on the software level), each engineering task is represented by a specific Webservices (WS). Complex ET formation is the process that is described by generation of web-services sequence (for the web-services that perform each partial ET included in the complex ET). Such Web-services sequence is called scenario. Thus, scenario represents complex engineering task (cET) from certain subject field, then each web service (scenario element) represents partial calculation task (pET).

Fig. 1 shows the interaction of basic concepts described above: complex engineering tasks, partial engineering tasks, web-services and scenarios.

Let us describe characteristic features of ETs that influence on their effective execution on the engineering knowledge portals:

- the decomposition of complex ET into partial ET,

- hierarchical subordination of ET,

- $\quad$ usage of the same partial ET in different complex ET,

- dependents of the ET execution order from their topic, parameters and characteristics,

- different directivity of ET.

One of the main problem with ET representation on knowledge portals is the description of their parameters (basic loading, winding radius, critical length, maximal diameter, etc.) that form the basis for partial ET integration.

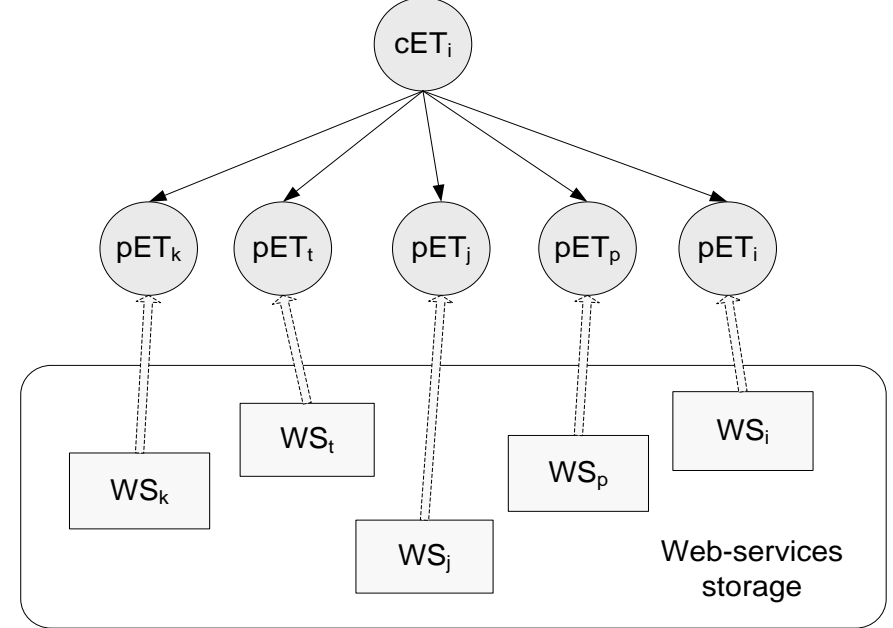

Scenario: $W S_{k} \rightarrow W S_{t} \rightarrow W S_{j} \rightarrow W S_{p} \rightarrow W S_{i}$

Fig. 1. Complex ET and elements that take part in its formation

Partial ETs set is stored in an independent repository that has no semantic and logical connections with the complex ET repository. Partial ET structure is similar to the complex ET structure and includes ET name and parameters. Fig. 2 represents the repository of partial ET and one complex ET, each of them has its own structure and set of parameters. 


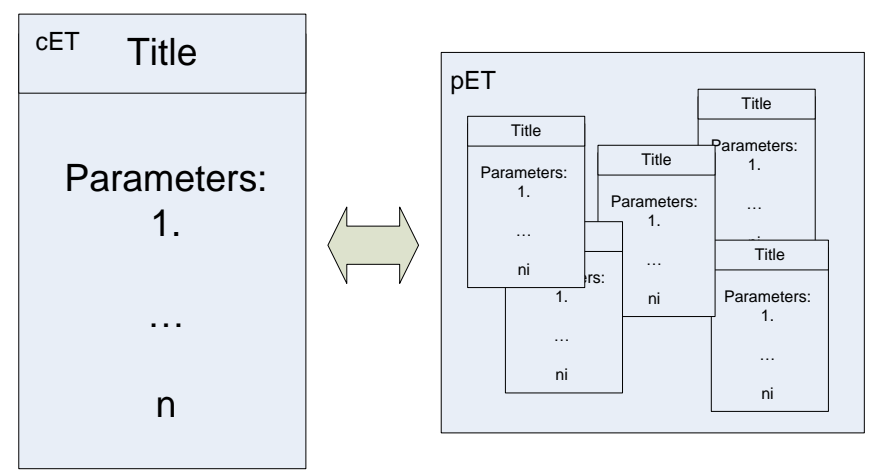

Fig. 2. Complex and partial ETs set

Search of the partial ET, to be included into the complex ET may be based on comparing of ET parameters, as complex ET includes only those partial ETs that have common parameters or parameter ranges of values overlapped. The formal description of partial and complex ETs sets is given below.

Let the set of complex ET to be:

$$
E T^{\mathrm{c}} \exists \mathrm{ET}_{\mathrm{k}}^{\mathrm{c}}, \mathrm{ET}_{\mathrm{k}}^{\mathrm{c}}=\left\langle\mathrm{T}_{\mathrm{k}}^{\mathrm{cET}}, \mathrm{p}_{\mathrm{k}}^{\mathrm{cET}}\right\rangle
$$

where

$\mathrm{ET}^{\mathrm{c}}-$ complex ETs set,

$\mathrm{ET}_{\mathrm{k}}^{\mathrm{c}}-k$-th ET from complex ETs set,

$\mathrm{T}_{\mathrm{k}}^{\mathrm{cET}}-$ title of $k$-th ET from complex ETs set,

$\mathrm{p}_{\mathrm{qk}}^{c E T}-q$-th parameter of $k$-th ET from complex ETs set.

Partial ETs set is:

where

$$
\mathrm{ET}^{p} \ni \mathrm{ET}_{1}^{c}, \mathrm{ET}_{1}^{p}=\left\langle\mathrm{T}_{1}^{p E T}, \mathrm{p}_{1}^{p E T}\right\rangle
$$

$\mathrm{ET}^{p}$ - partial ETs set,

$E T_{1}^{p}-l$-th ET from partial ETs set,

$\mathrm{T}_{1}^{p E T}$ - title of $l$-th ET from partial ETs set,

$\mathrm{p}_{\mathrm{tl}}^{p E T}-t$-th parameter of $l$-th ET from partial ETs set.

As discussed approach deals with engineering tasks of knowledge portal, assume that each ET is uniquely identified by set of its parameters. It means that two ET with the same parameters could not have a different semantic in what they do and meanings of partial and complex ETs parameters could be used for partial ET integration into the complex one. Using such formal description, specific method can be used to automate ET composition.

\section{THE ALGEBRAIC SYSTEM OF ENGINEERING TASKS}

ETs formal algebraic system allows to use formal algebraic structures for ETs elements description as well as to describe operations with ETs elements when engineering knowledge portal is functioning.

Such formal description will allow complex ET correct and fast composition.

The algebraic model of ETs is represented by a set of certain type operations on a stored set of information and computing resources $[36,37]$.

The elements of the proposed ETs algebraic model are represented below.

Objects. Objects are the basic elements of the algebra on which all operations of algebra are conducted. In ETs algebraic model as the variety of objects set of ETs are mentioned.

Data. Elements that are given to the input and output of the system represent the data of algebra. In ETs algebraic model, such objects can be used as data: different constants, variables, results of operations, etc. On the physical level, the data are represented by the values of various parameters and characteristics of the subject domain, formulas, values boundaries, as well as partial services and computational procedures that rep- resent calculation tasks [38].

Operations. Set of operations in the ETs algebraic model is divided into two subsets: subset of simple operations and subset of complex operations [39].

Simple operations include elementary algebra operations according to the definition, operations on set (given in the set theory) and logical operations defined by algebra of relations.

Complex operations are:

- the operation of parallel connection Opc that describes the process of ETs connection with disjunction logical operation;

- the operation of serial connection Osc that describes the process of ETs connection with conjunction logical operation;

- $\quad$ operation of logical composition Oc that allows to connect ETs by means of known relationship between them and interim ET;

- operation of inversion Oi that provides a replacement of the ETs order to the opposite during the connection of partial Et into a complex one;

- matching operation Om that allows to connect ETs that are not connected explicitly using some parameters.

More detailed description of proposed algebraic system is given in [40].

\section{THE METHOD OF ENGINEERING TASKS COMPOSITION}

Proposed in section 3 and 4 formal description and algebraic system allow to develop the method of ETs composition, allowing correctly and dynamically generate a sequence of partial ETs that are in need to be included into the complex ET when the portal is functioning.

ETs composition using proposed method can be presented as an ordered tree. Whereas the ordered tree is a tree with a root, which defines the order of child nodes, the usage of ordered tree for representation of complex ET composition process will specify the sequence of partial ETs included in a complex one execution.

The suggested algorithm for the method of ETs composition is represented on Fig.3. Fig. 4 shows the tree that is formed using proposed method. The root node shows complex ET, tree leaves are partial ETs.

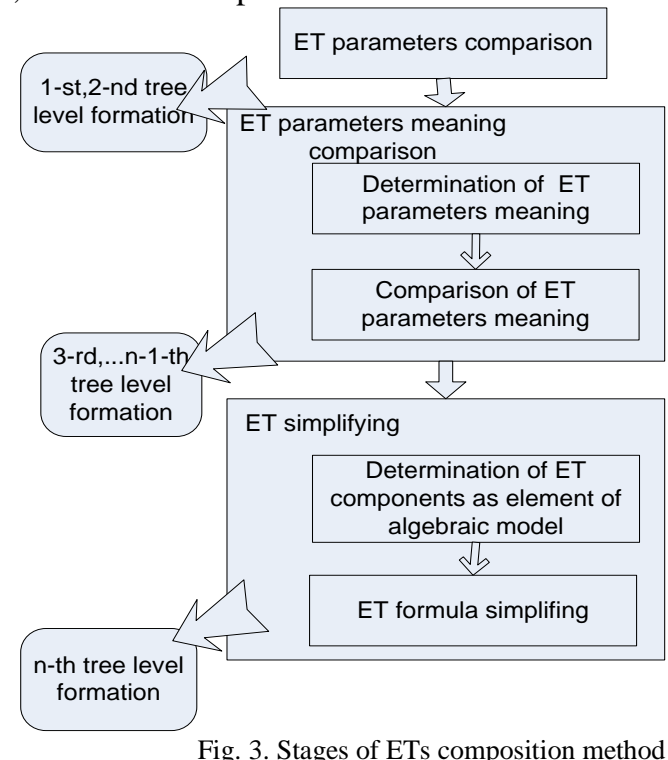

Fig. 3. Stages of ETs composition method 


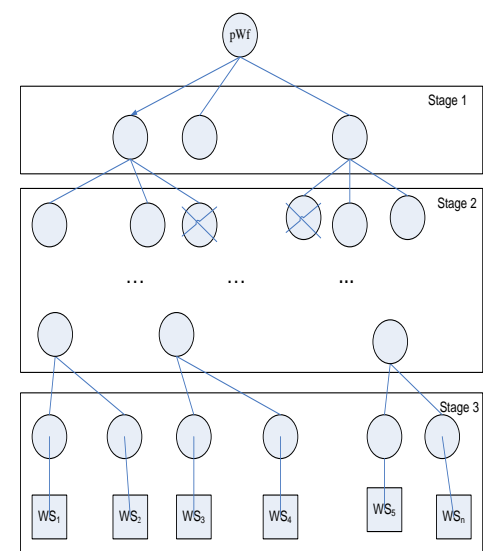

Fig. 4. Complex ET tree

Stage 1. Comparing ET parameters.

At the first stage it is in need to discard from the set $\mathrm{ET}^{p}$ those ETs, for which $\mathrm{p}_{\mathrm{tl}}^{p E T}$ is not equal to $\mathrm{p}_{\mathrm{qk}}^{c E T}$. It is in need to compare and select those partial ETs which parameters match the complex ET parameters. It is necessary to analyze the partial ETs set and choose from it those ETs, which parameters match the complex ET parameters according to the rule:

$$
\mathrm{p}_{\mathrm{d}}^{\mathrm{cET}}=\bigcup_{\mathrm{i}}^{\mathrm{m}} \mathrm{P}_{\mathrm{i}}^{p E T}
$$

whereby possible the fulfilment:

where $i, j=\overline{1, m}$

$$
\mathrm{p}_{\mathrm{i}}^{p E T} \cap \mathrm{p}_{\mathrm{j}}^{p E T}
$$

The result is a subset of partial ET set satisfying the rule (4) and complex ET tree with second level tops, which are elements of this subset.

Stage 2. Checking ETs parameters values.

It is necessary to compare meanings of $\mathrm{p}_{\mathrm{tl}}^{E T}$ to cut the subset of partial ETs, that has common parameters but their meanings are not equal. The comparison is done based on the occurrences or equality of these parameters meaning range of values.

Thus, on the second stage ETs parameters values from partial ETs set, formed on the first stage, are compared to complex ET corresponding parameters. The comparison is held due to the rule:

$$
\mathrm{M}\left(\mathrm{p}_{\mathrm{il}}^{\mathrm{ET}}\right)<>^{\mathrm{M}}\left(\mathrm{p}_{\mathrm{jr}}^{E T}\right)
$$

under the condition that $\mathrm{p}_{\mathrm{il}}^{E T}=\mathrm{p}_{\mathrm{jr}}^{E T}$

Stage 3. Simplifying ET formula.

The third stage operates with specific rules and operations to represent the formula of ET (described using proposed algebraic model) in minimized form. Such simplifications rules are based on properties of operations [41]. Operations properties form the basis for the main identities of algebraic model underlying the formulas transformation for the purpose of simplification or reduction to a certain form.

The resulting complex ET tree can be saved in the knowledge base as a pattern for further use with the possibility of its modification. It can be modified using standard operations on trees.

Presenting the complex engineering tasks in the form of tree structure allows to process each node of the tree, which represents a different partial ET, simultaneously, avoiding tree branches, which are independent of each other, which will minimize time for complex ET composition.

\section{EXAMPLE OF THE COMPLEX ENGINEERING TASK COMPOSITION}

To confirm the efficiency of the proposed method the test group of complex ETs for problem domain "Strength of materials" was selected (Tab. 1), which consists of five complex ETs that includes 183 partial ETs.

Table 1

\begin{tabular}{|c|c|c|}
\hline № & The title of complex ET & $\begin{array}{c}\text { The amount of } \\
\text { partial ET in a } \\
\text { complex ET }\end{array}$ \\
\hline 1 & $\begin{array}{c}\text { Calculation of the strength for the power compo- } \\
\text { nents of magnetic systems }\end{array}$ & 56 \\
\hline 2 & $\begin{array}{c}\text { Calculation of the strength for equipment and } \\
\text { pipelines of nuclear power systems }\end{array}$ & 33 \\
\hline 3 & $\begin{array}{c}\text { ITER Structural Design Criteria for magnetic com- } \\
\text { ponents }\end{array}$ & 28 \\
\hline 4 & $\begin{array}{c}\text { Magnet DDD 1.1-1.3. Magnet System Design } \\
\text { Criteria }\end{array}$ & 18 \\
\hline 5 & $\begin{array}{c}\text { Calculation of the strength for the elements of } \\
\text { equipment and pipelines of ship nuclear steam } \\
\text { generating systems with a water reactors }\end{array}$ & \\
\hline
\end{tabular}

Let us show the example of complex ET from the test group formation using proposed method. This ET is «Calculation of the strength for the power components of magnetic systems». At the 1-st stage the analysis of partial ETs set was done. It was in need to choose from this set only those partial ETs that satisfies condition (5). Such ETs are:

- calculation of basic parameters - $E T_{1}^{p}$;

- verifying calculation $-E T_{2}^{p}$;

- $\quad$ strength calculation - $E T_{3}^{p}$,

- critical constant calculation - $E T_{4}^{p}$;

- resistance calculation - $E T_{5}^{p}$;

- dimensioning of reactors coil corps with arched slit $E T_{6}^{p}$

- calculation of reducing the strength of welds -.ET

- calculation of flanges, rings and fasteners $-E T_{8}^{p}$.

- calculation of static strength $-E T_{9}^{p}$.

- calculation of stability $-E T_{10}^{p}$.

- calculation of cyclic strength $-E T_{11}^{p}$.

- calculation of crack resistance $-E T_{12}^{p}$.

- calculation of voltage variation $-E T_{13}^{p}$

- calculation of seismic impacts $-E T_{14}^{p}$.

- calculation of the vibration strength $-E T_{15}^{p}$.

- calculation of the limiting value of deformation $-E T_{16}^{p}$.

- calculation of the vibration stability $-E T_{17}^{p}$

Formed complex ET tree is shown on Fig. 5. The root node show complex ET: $\mathrm{ET}_{1}^{\mathrm{C}}$ - "Calculation of the strength for the power components of magnetic systems", intermediate nodes are ETs: $\mathrm{ET}_{1}^{p}$ - "Calculation of basic parameters" and $\mathrm{ET}_{2}^{p}-$ "Verifying calculation", leaves are the rest described ETs. 


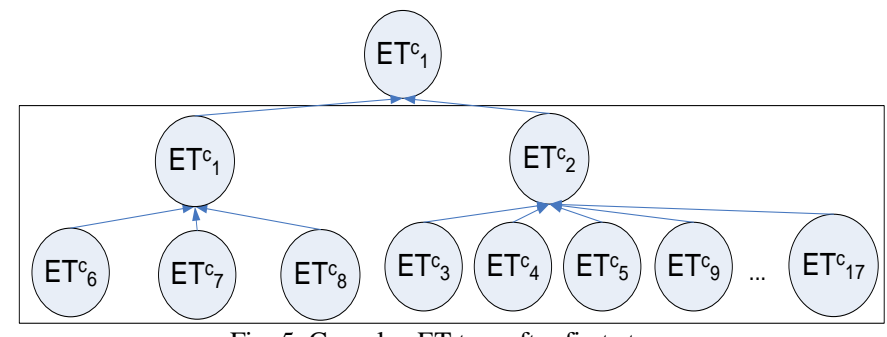

Fig. 5. Complex ET tree after first stage

At 2-nd stage, the comparison of ETs parameters meanings was held according to the rule (6). Some partial ETs were discarded from the set formed on the first stage, as some of their parameters are out of range of values with values of the same parameters of general ET. The following ETs were discarded:

- critical constant calculation - $E T_{4}^{p}$;

- calculation of stability $-E T_{10}^{p}$.

- calculation of cyclic strength $-E T_{11}^{p}$.

- calculation of voltage variation $-E T_{13}^{p}$

- calculation of the vibration stability $-E T_{17}^{p}$

Complex ET tree after 2-nd stage is shown on Fig. 6. Tree includes the same nodes and leaves as tree represented on Fig. 6, but $E T_{4}^{p}, E T_{10}^{p}, E T_{11}^{p}, E T_{13}^{p}, E T_{17}^{p}$ - were removed from the tree as the result of the 2-nd stage.

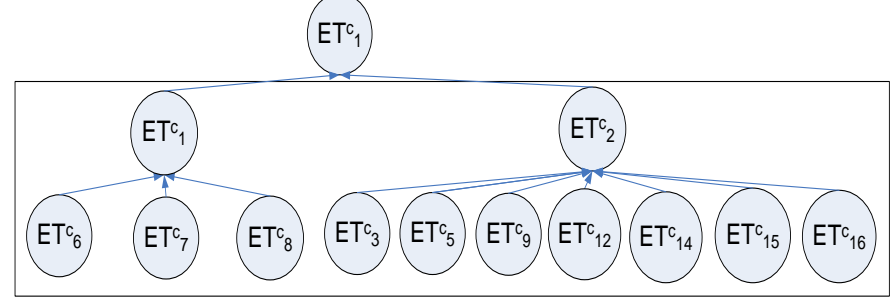

Fig. 6. Complex ET tree after 2-d stage

The estimation of the time required to develop scenarios for complex engineering tasks was made. The estimation was made for static way of partial ET composition and for their composition with the help of proposed method (Fig. 7).

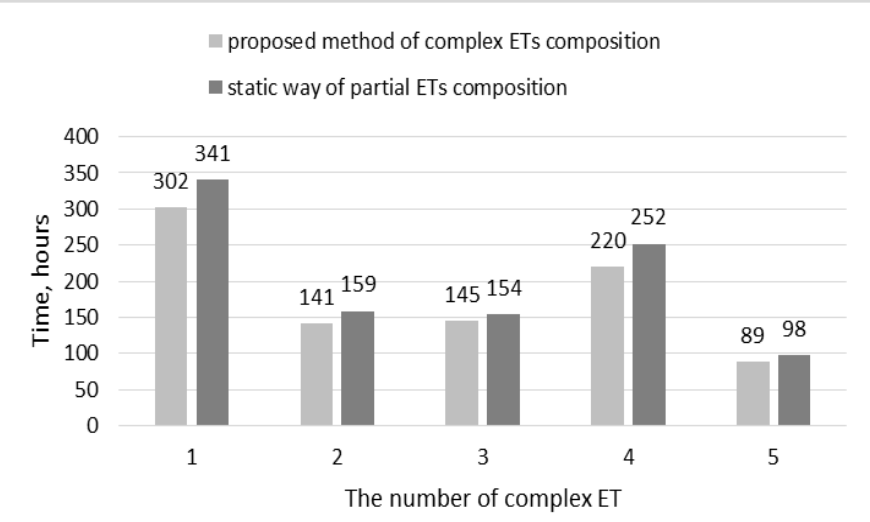

Fig. 7. Estimation of the complex ET composition time

Time needed for services development with the help of static way of partial ET composition is 1004 hours and with the help of proposed method is 897 hours. Thus, the proposed method allowed to reduce the time for scenario for complex engineering task development by $11 \%$.

\section{CONCLUSIONS}

The paper presents an approach to engineering task composition on engineering knowledge portals. The approach is passed on formal algebraic system and method of engineering tasks composition. Formal algebraic system allows to set the context-independent formal structures for engineering tasks elements description.

The proposed method of engineering tasks composition allows to integrate partial engineering tasks into a complex one on engineering knowledge portal, performed on user's request. It also allows to represent the process of complex engineering task composition as ordered tree that improves the efficiency of their performance through parallel processing of independent tree branches.

Future work is aimed on implementation of suggested approach to different types of complex engineering calculation tasks. This will allow to approve its applicability and efficiency on real world scenarios. Developed software tool will be tested and verified on real world scenarios when engineering knowledge portals are developed.

Quantitative evaluation of the proposed approach and tool efficiency will be obtained for different subject domains: the average time of engineering task composition, engineering tasks correctness and quality will be validated.

\section{REFERENCES}

1. Tony Byrne, Real Story Group Blog - Digital workplace and enterprise architecture: two sides to same coin (2012) $\mathrm{http}: / / \mathrm{www}$. realstorygroup.com/Blog/2311-Digital-workplaceand-enterprise-architecture-two-sides-to-same-coin

2. Microsoft SharePoint 2010 Capabilites: http://sharepoint.microsoft.com/ru$\mathrm{ru} /$ product/capabilities/composites/Pages/default.aspx.

3. IBM WebServers overview: http://www01.ibm.com/software/webservers/appserv/was/.

4. SAP Portal Collaboration content management: http://www.sap.com/solutions/technology/collaboration-contentmanagement/enterprise-portals/index.epx.

5. Choosing portal platform// Overview of modern platforms: http://www.intranetno.ru/news/9527/.

6. John F. Sowa The role of logic and ontology in language and reasoning, Chapter 11 of Theory and Applications of Ontology: Philosophical Perspectives, edited by R. Poli \& J. Seibt, Berlin: Springer, 2010, pp. 231-263.

7. F. Bobillo, U. Straccia. Aggregation Operators for Fuzzy Ontologies. Applied Soft Computing 13(9):3816-3830, 2013

8. Guarino N., Guizzardi G. In Defense of Ontological Foundations for Conceptual Modeling. In: Scandinavian Journal of Information Systems, vol. 18(1) pp. 115 - 126. 2006.

9. D. Movshovitz-Attias and W.W. Cohen. 2012. Boot-strapping biomedical ontologies for scientific text using nell. Technical report, Carnegie Mellon University, CMU-ML-12-101.

10. Zagorulko U.A. and others. An approach to portals of scientific knowledge development // Avtometriya. 2008. V. 44. № 1. P. $100-110$.

11. Gavrilova T.A. Horoshevskyi V.F. Knowledge bases of intellectual systems. - S-P.: Piter, 2000

12. Kolb D.G. Web-oriented realization of semantic models for intellectual systems // Proceedings of scientific conference "«Open Semantic Technologies for Intelligent Systems (OSTIS2012)", - Minsk, 2012. - P. 111-122.

13. Globa L. Method of heterogeneous information resources structuring and systematizing for Internet portals development / Globa L., Novogrudska R., Oriekhov O. // In: IEEE EUROCON, pp. 319-326 (2013).

14. Zagorulko U.A. and others. The technology of scientific portals development: experience of application, problems and prospects. Proceedings of international scientific conference «SWCtechnique and telecommunication technologies» (Crymico' 2011). - Sevastopol: Veber, 2011. - P. 51-54.

15. Pukhkaiev, D., Kot, T., Globa, L., Schill, A.: A novel SLAaware approach for web service composition. In: IEEE EUROCON, pp. 327--334 (2013) 
16. S. Dustdar, and W. Schreiner, "A survey on web services composition," in Int. J. Web and Grid Services, 2005, vol. 1, No. 1, p.1-30.

17. Sheng, Q. Z, Qiao, X., Vasilakos, A. V., Szabo, C., Boume, S., $\& \mathrm{Xu}, \mathrm{X}$. (2014). Web services composition: A decade's overview. Information Sciences 280, 218-238.

18. Moghaddam, M., \& Davis, J. G. (2014). Service selection in web service composition: A comparative review of existing approaches. In: Web Services Foundations (pp. 321-346). Springer New York.

19. Shehu, U., Epiphaniou, G., \& Safdar, G. A. (2014). A survey of QoS-aware web service composition techniques. International Journal of Computer Applications.

20. Upadhyaya, B. (2014). Composing Heterogeneous Services from End Users' Perspective.

21. McIlraith, S. A., Son, T. C., \& Zeng, H. (2001). Semantic web services. IEEE intelligent systems, (2), 46-53.

22. Medjahed, B., \& Bouguettaya, A. (2011). Service composition for the Semantic Web. Springer Science \& Business Media.

23. Martin, D., Paolucci, M., McIlraith, S., Burstein, M., McDermott, D., McGuinness, D., ... \& Srinivasan, N. (2004). Bringing semantics to web services: The OWL-S approach. In Semantic Web Services and Web Process Composition (pp. 26-42). Springer Berlin Heidelberg.

24. Miller, J., Verma, K., Rajasekaran, P., Sheth, A., Aggarwal, R., \& Sivashanmugam, K. (2004). Wsdl-s: Adding semantics to wsdl-white paper.LSDIS Lab, University of Georgia, Georgia, USA.

25. De Oliveira Jr, F. G. A., \& de Oliveira, J. M. P. (2011). QoSbased Approach for Dynamic Web Service Composition. J. UCS, 17(5), 712-741.

26. Papazoglou, M. P., \& Van Den Heuvel, W. J. (2007). Service oriented architectures: approaches, technologies and research issues. The VLDB journal, 16(3), 389-415.

27. Hatzi, O., Vrakas, D., Nikolaidou, M., Bassiliades, N., Anagnostopoulos, D., \& Vlahavas, I. (2012).

28. An integrated approach to automated semantic web service composition through planning. Services Computing, IEEE Transactions on, 5(3), 319-332.
29. Ngan, L. D., \& Kanagasabai, R. (2013). Semantic Web service discovery: stateof-the-art and research challenges. Personal and ubiquitous computing, 17(8), 1741-1752

30. E.M. Maximilien and M.P. Singh., "A framework and ontology for dynamic web services selection," IEEE Internet Computing, vol. 8, pp. 84-93, Sep./Oct. 2004.

31. M. B. Blake, and D. J. Cummings, "Workflow Composition of Service-Level Agreements," in Proceedings of IEEE International Conference on Services Computing (SCC), July 2007, p.138-145.

32. A. Andrieux, K. Czajkowski, A. Dan, K. Keahey, H. Ludwig, T. Nakata, J. Pruyne, J. Rofrano, S. Tuecke, and M. Xu. (2007) Web Services Agreement Specification (WS-Agreement) Online]. Available:http://www.ogf.org/documents/GFD.107.pdf.

33. J. Cardoso, and A. Sheth, Semantic Web Services, Processes and Applications, Springer, 2006.

34. Norms for the strength calculation for the power components of magnetic systems. - Kyiv: ISP, 1984. $-73 \mathrm{c}$.

35. Norms for the strength calculation for equipment and pipelines of nuclear power systems - M.: Energoatomizdat, 1989. - $525 \mathrm{c}$.

36. Globa L.S., Novogrudska R.L., The development of engineering calculations for knowledge portals // Proceedings of scientific conference «Open Semantic Technologies for Intelligent Systems (OSTIS - 2014)». - Minsk, 2012. - P. 137-143

37. Pavlov A.A., Telenyk S.F. Informational technologies and algorithmization in management. - K.:Tehnika, 2002. - 344 p.

38. Shahovska N.B. Data space in sphere of scientific research // Modeling and information technologies. -2008 . - № 45. - P. $132-140$

39. John Dwyer. An Introduction to Discrete Mathematics for Business \& Computing. ISBN 978-1-907934-00-1. (2010)

40. Globa L.S., Novogrudska R.L., An approach to formal system for knowledge portals development, Ontology of designing. 2014. - №2(11). - ISSN 2223-9537- P.40-59

41. Kenneth H. Rosen. Discrete Mathematics: And Its Applications. McGraw-Hill College. ISBN 978-0-07-288008-3. (2007) 